EPJ Web of Conferences 95,01008 (2015)

DOI: $10.1051 /$ epjconf/20159501008

C) Owned by the authors, published by EDP Sciences, 2015

\title{
Recent highlights from BESIII: XYZ states
}

\author{
W. Kühn ${ }^{1, a}$, on behalf of the BESIII Collaboration \\ ${ }^{1}$ Justus-Liebig-University Giessen, Germany
}

\begin{abstract}
In this contribution we review recent results from the BESIII experiment. We focus on the investigation of XYZ states and the discovery of new charged charmonium - like structures. Two isospin triplets Z(3900) and Z(4020) have been discovered, decaying into $\pi^{ \pm} J / \psi$ and $\pi^{ \pm} h_{c}$, respectively. Structures with compatible parameters have been found decaying to $D \bar{D}^{*}$ and $D^{*} \bar{D} *$, respectively, suggesting that these structures might be of the same origin. Furthermore, we have investigated the transition $Y(4260) \rightarrow \gamma X(3872)$. This is the first observation of a radiative transition between XYZ states.
\end{abstract}

\section{Introduction}

Hadrons are fundamental states bound by the strong interaction. Until recently, only two classes of hadrons were firmly established experimentally: baryons (anti-baryons), consisting of 3 quarks (anti-quarks) and mesons, consisting of quark / antiquark combinations. Hadrons are integer-charged color singlets, bound by the exchange of gluons carrying color charge, as described by Quantum Chromodynamics (QCD).

QCD is a fundamental gauge theory based on $S U(3)_{\text {color }}$ symmetry. The exchange bosons, the gluons, carry color charge. As a consequence, a complex phenomenology emerges. The coupling constant $\alpha_{s}$ is strongly momentum dependent. Two regimes exist: (i) the perturbative regime at large momentum transfers / small distances and (ii) the non-perturbative regime at small momentum transfers / large distances. Perturbation theory is extremely successful to describe the properties of hard scattering processes. In the non-perturbative regime, perturbation theory breaks down but Lattice QCD and effective field theories can be applied.

It should be noted that hadronic uncertainties can have considerable impact on the sensitivity of searches for physics beyond the standard model. Hence, an improved understanding of nonperturbative QCD is important and our level of understanding should be tested by confronting experiment and theory.

There is no a-priori reason following from QCD why only Mesons and Baryons should exist as bound states of quarks. Objects such as di-baryons (6 quark states), tetra quarks (two quark / two anti -quark states), hybrids (quark / anti-quark / gluon states) or glue balls (states consisting only of gluons) have been predicted to exist. So far, however, no compelling undisputed evidence for the existence of such exotic states has been found by experiments.

\footnotetext{
ae-mail: W.Kuehn@physik.uni-giessen.de
} 
In the light meson sector, many new states have been discovered that have peculiar properties not fitting to the known meson nonets. Here, various candidates for exotic states have been found. Their analysis is complicated by the fact that these states are broad and mixing can occur. Furthermore, states with exotic quantum numbers, i.e. quantum numbers that cannot be ascribed to a $q \bar{q}$ system have been found, representing potential candidates for hybrid states or mesonic molecules.

In the heavy quark sector, the situation is more favorable. The decay of heavy quarkonia into light mesons is OZI suppressed. As a consequence, states are narrow and well separated. Furthermore, the spectroscopy of these states can be well described by various theoretical approaches such as static potential models, effective field theories and lattice QCD.

Until about 10 years ago, the spectroscopy of heavy quarkonia was believed to be well understood. Most states below the open charm and open beauty thresholds had been found and their properties could be reasonably well understood in terms of theory. A wealth of information has been extracted leading to a better understanding of the QCD potential, binding quarks and anti-quarks in heavy quarkonia. The striking similarity to the spectroscopy of positronium lead to the conclusion that the QCD potential exhibits a Coulomb - like shape at small distances.

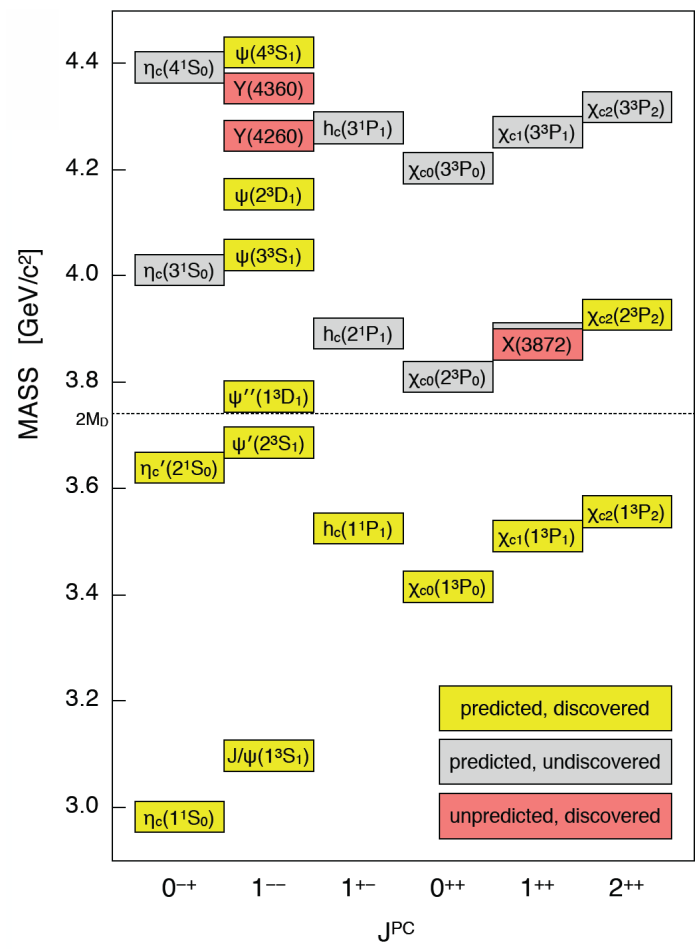

Figure 1. Spectroscopy of charmonia and charmonium-like states. States marked in yellow are conventional charmonium states that were experimentally established and predicted by theory. States marked in grey are predicted but have not been found by experiment up to now. States marked in red were not predicted $\mathrm{b}$ theory but found by experiment. Figure courtesy of Ryan Mitchell, Indiana University.

Above the open charm threshold, the situation is more complex. Some of the predicted charmonium states have been found, many have not yet been observed. Since 2003, many new unpredicted states have been found with properties that are not consistent with those of conventional charmonium states.

These states were called XYZ states since their nature is not understood and thus the existing naming scheme for mesons is not appropriate. Here, $\mathrm{X}$ states refer to neutral states containing $c \bar{c}$ with quantum numbers different from $J^{P C}=1^{--}$. Y-states refer to $1^{--}$states containing $c \bar{c}$ with properties that exclude their interpretation as conventional charmonia. Z-states are charged charmonium-like 
states. Such states, if they exist, must contain at least two quarks and two anti-quarks, among them a $c \bar{c}$ pair. All these these states have peculiar properties and do not fit into the established spectroscopy above the open charm threshold.

The theoretical interpretation of the XYZ states is not clear. Many explanations have been proposed including hybrids, tetra quarks, hadronic molecules, re-scattering effects near open charm thresholds, hydro-charmonium and other models. For an overview, see ref. [4]

Fig. 1 shows an overview of the charmonium system. States marked in yellow are conventional charmonium states that were experimentally established and predicted by theory. States marked in grey are predicted but have not been found by experiment up to now. States marked in red were not predicted by theory but found by experiment.

The $\mathrm{B}$ factories (BaBar, Belle) have produced very large numbers of heavy quarkonia. Here, the first XYZ - states were discovered. Fig 2 (left) shows the observertion of the X(3872) state by Belle in $\mathrm{B}$ decays [1]. An extremely narrow $(\Gamma<1.2 \mathrm{MeV})$ resonance at $3872 \mathrm{MeV}$ is visible, decaying into $J / \psi \pi^{+} \pi^{-}$. A peculiar feature of the $\mathrm{X}(3872)$ is its location right at the $D_{0}{\overline{D_{0}}}^{*}$ threshold. This state has been confirmed by many other experiments.

Recently, LHCb has determined the quantum numbers of the $\mathrm{X}(3872)$ as $J^{P C}=1^{++}$[3]. The narrow width of this state is remarkable, in particular when compared to the $\psi^{\prime \prime}(3770) 1^{--}$charmonium state $(\Gamma \sim 27.2 \mathrm{MeV})$. Fig. 2 (right) sBESIIIhows the discovery of the Y(4260) state by BaBar [2],
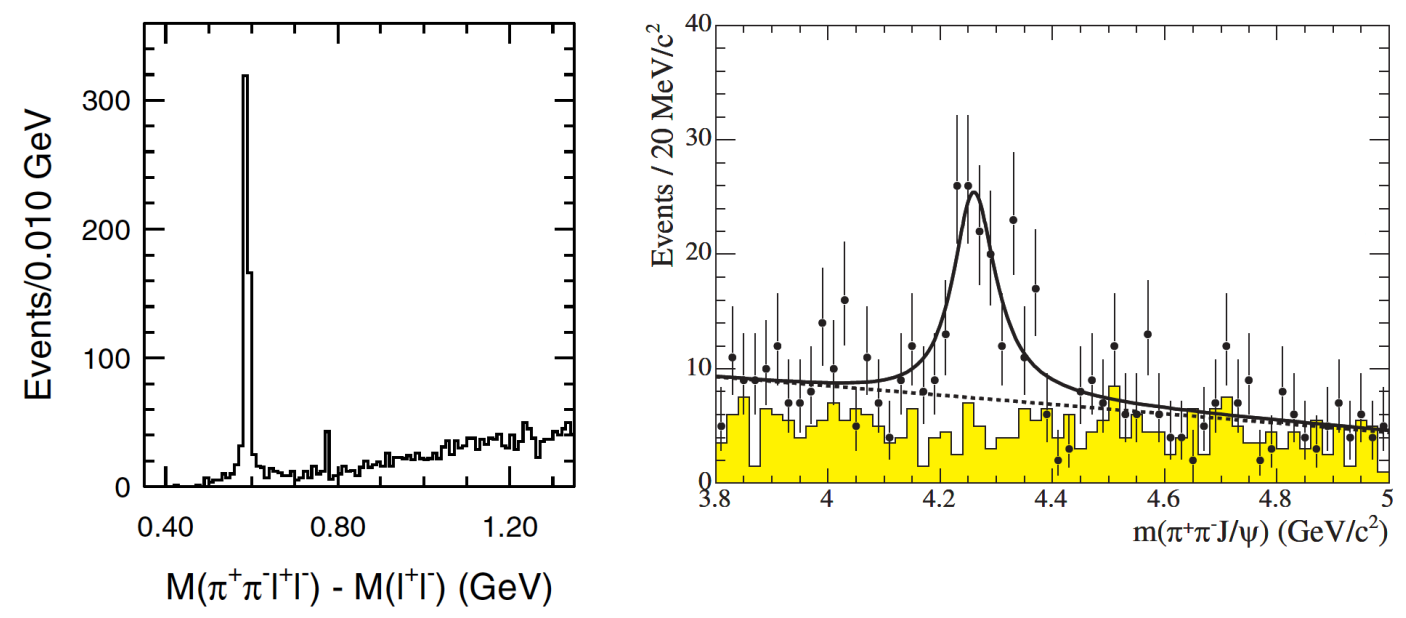

Figure 2. (left) Discovery of the X(3872) by Belle [1]. For properly selected $J / \psi \rightarrow e^{+} e^{-} / \mu^{+} \mu^{-}$events from $B^{ \pm} \rightarrow K^{ \pm} \pi^{+} \pi^{-} J \psi$, the distribution of the difference of invariant masses of $\pi^{+} \pi^{-} J \psi$ and the lepton pair is shown. The $\mathrm{X}(3872)$ is visible as a narrow structure, in addition to a prominent $\psi^{\prime \prime}$ peak; (right): discovery of the $\mathrm{Y}(4260)$ in initial state radiation by BaBar [2].

using ISR radiation. This state has a strong decay mode into $J / \psi \pi^{+} \pi^{-}$. In contrast, conventional $1^{--}$ charmonium states above the open charm threshold decay almost exclusively into final states with open charm. The Y(4260) has been observed by many experiments.

More recently, two new experimental facilities, the BESIII experiment at IHEP Beijing and the $\mathrm{LHCb}$ experiment at CERN are contributing to the field. In this paper, we will focus on recent results on XYZ states from BESIII including the discovery of new charged charmonium - like structures $\mathrm{Z}(3900)$ and $\mathrm{Z}(4020)$ as well as their neutral partners. Furthermore, we discuss the observation of a 
radiative transition between $\mathrm{XYZ}$ states. Here, we exploit the fact that BESIII can populate the exotic $1^{--} \mathrm{Y}$ states directly via $e^{+} e^{-}$annihilation into a virtual photon.

\section{BEPCII Collider and BESIII detector}

The BESIII experiment[5] is located at the BEPCII $e^{+} e^{-}$collider at the Institute of High Energy Physics (IHEP) Beijing. The energy range from $\sqrt{\mathrm{s}}=2 \mathrm{GeV}$ up to $4.6 \mathrm{GeV}$ is providing access to physics in the $\tau$-charm energy region. double ring $e^{+} e^{-}$collider is designed for a peak luminosity

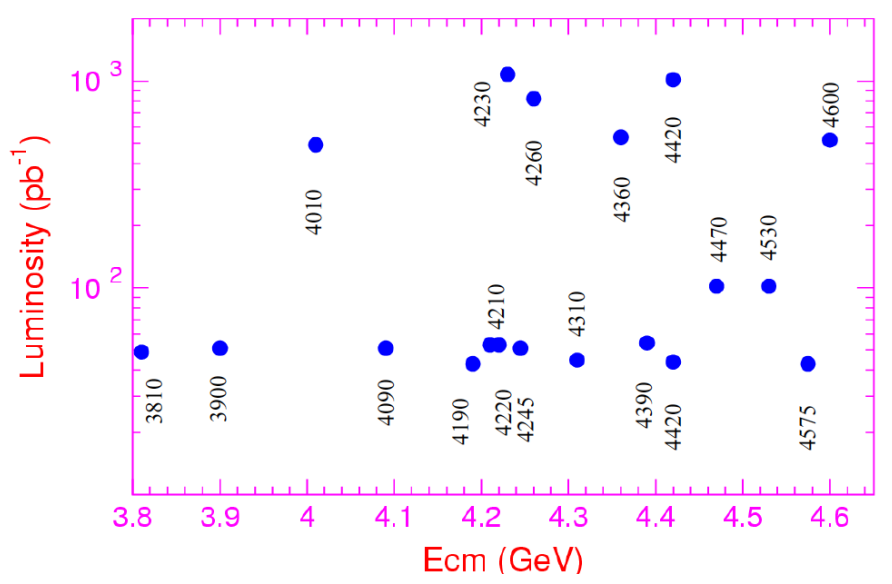

Figure 3. Integrated luminosity at various energy points of relevance for XYZ physics taken with BESIII in 2012 and 2013

of $10^{33} \mathrm{~cm}^{-2} \mathrm{~s}^{-1}$ at the $\psi(3770)$ resonance. The detector has an angular acceptance of about $93 \%$ of $4 \pi$ and features the following major components: (1) a helium gas based drift chamber (MDC) with 43 layers, providing a single wire spatial resolution of 135 micron, a dE/dx resolution of better than $6 \%$, and a momentum resolution of $\sim 0.5 \%$ for charged particles with momenta of $1 \mathrm{GeV} / \mathrm{c}$ in a 1 Tesla magnetic field produced by a superconducting solenoid. (2) The time-of-flight system (TOF) system for particle identification, consisting of a two-layer structure in the barrel and a one layer structure in the endcap region. Plastic scintillators provide a time resolution of $80 \mathrm{ps}(110 \mathrm{ps})$ in the barrel (endcap) system allowing a pion/kaon separation at a 95\% C.L. up to about $1 \mathrm{GeV} / \mathrm{c}$. (3) The electromagnetic calorimeter (EMC) surrounding the MDC and the TOF system, consisting of 6240 Th doped CsI- crystals with an energy resolution of $2.5 \%(5.0 \%)$ and a position resolution of $6 \mathrm{~mm}(9 \mathrm{~mm})$ for photons with an energy of $1 \mathrm{GeV}$ in the barrel (endcap) part. (4) The muon chamber system, embedded in the magnetic flux return, consisting of 9 (8) layers of RPC in the barrel (endcap) region with a spatial resolution of $2 \mathrm{~cm}$.

For the purpose of XYZ physics, data sets with a total integrated luminosity of $5 / \mathrm{fb}$ have been collected (see Fig. 3. It should be noted that BESIII has a very rich physics program, including light hadron spectroscopy, charmonium physics, open charm physics, $\tau$ lepton physics, precision measurement of the R value (important input to BSM searches) and other topics that are not covered in this contribution. 


\section{New XYZ states at BESIII}

The field of XYZ states has been pioneered by the B factory experiments BaBar and Belle both using $\mathrm{B}$ decays and ISR. BESIII, however, can directly populate exotic Y states with quantum numbers $1^{--}$ and collect data sets with large integrated luminosity, focussing on hadronic decays to charmonium states. The first discovery [6] was made when looking for $e^{+} e^{-} \rightarrow \pi^{+} \pi^{-} J / \psi$ at center of mass energies of $4230 \mathrm{MeV}, 4260 \mathrm{MeV}$ and $4360 \mathrm{MeV}$, respectively. Fig. 4 (left) shows the invariant mass
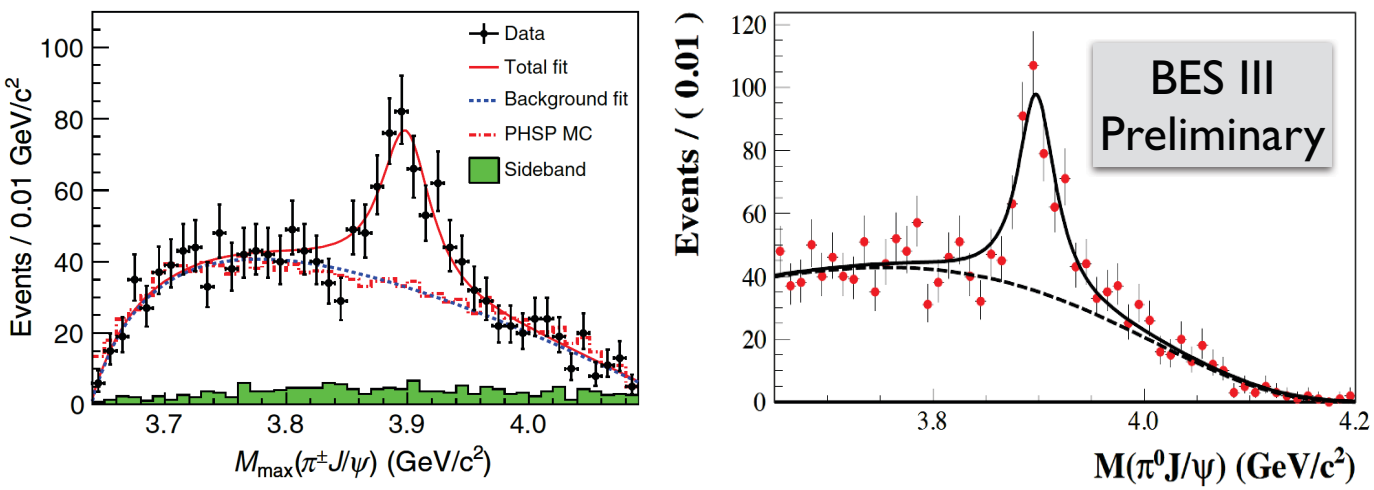

Figure 4. (left): Dots with error bars represent the measured $\pi^{ \pm} J / \psi$ mass distribution; the red solid curve shows the total fit; the blue dotted curve represents the background from the fit; the red dotted-dashed histogram shows the result of a phase space (PHSP) MC simulation; the green shaded histogram shows the normalized $J / \psi$ sideband events. (right): $\pi^{0} J / \psi$ invariant mass distribution with the total fit (solid line) and the background fit component (dashed line) superimposed.
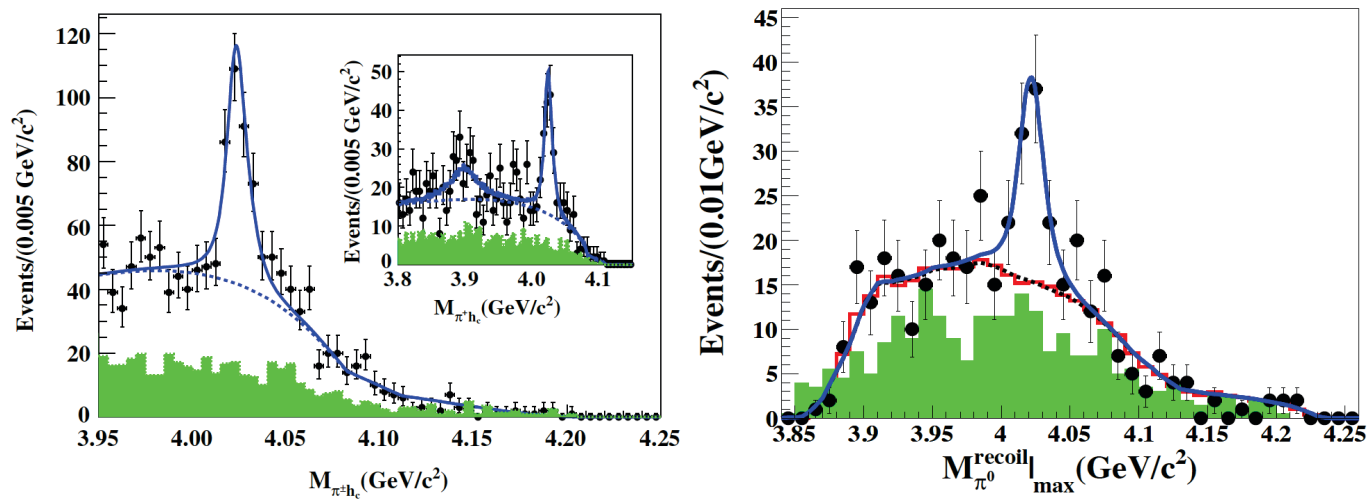

Figure 5. Sum of simultaneous fits to the invariant mass distributions of $\pi^{ \pm} h_{c}$ (left) and $\pi^{0} h_{c}$ (right) at $\sqrt{s}=4.23$, $4.26,4.36 \mathrm{GeV}$; the inset shows a simultaneous fit to the $\pi^{ \pm} h_{c}$ distributions at 4.23 and $4.26 \mathrm{GeV}$, including the Z(3900) and Z(4020). Dots with error bars are data; shaded histograms are the normalized sideband background; the solid curves show the total fit, and the dotted curves display the backgrounds from the fit.

distribution of $\pi^{ \pm} J / \psi$ pairs. A prominent structure, the $Z_{c}(3900)$, can be fitted with a resonant line 
shape with a mass and width of $(3899.0 \pm 3.6 \pm 4.9) \mathrm{MeV} / \mathrm{c}^{2}$ and $(46 \pm 10 \pm 20) \mathrm{MeV}$, respectively. If the $Z_{c}(3900)$ correspond to a new hadronic state containing $c \bar{c}$, its electric charge implies that it must contain at least four quarks. The observation of the $Z_{c}(3900)$ has been confirmed by Belle [7] in ISR as well as by an analysis of CLEOc data[8]. Thus, this is the first observation of a charged charmonium-like structure that has been confirmed by multiple experiments.

\begin{tabular}{|c|c|c|c|c|c|c|}
\hline & $D^{+\%}$ & $D^{0}$ & $D^{* / \%}$ & $D^{* 0}$ & $D_{1}(2420)^{\circ}$ \\
\hline & 1870 & 1865 & 2010 & 2007 & 2421 \\
\hline$D^{+\%}$ & 1870 & 3739 & 3734 & 3880 & 3877 & 4291 \\
\hline$D^{0}$ & 1865 & 3734 & 3730 & 3875 & 3872 & 4286 \\
\hline$D^{*+/}$ & 2010 & 3880 & 3875 & 4021 & 4017 & 4432 \\
\hline$D^{* 0}$ & 2007 & 3877 & 3872 & 4017 & 4014 & 4428 \\
\hline$D_{1}(2420)^{0}$ & 2421 & 4291 & 4286 & 4432 & 4428 & 4843 \\
\hline
\end{tabular}

Figure 6. Thresholds for decay into pairs of open charm mesons values in $\mathrm{MeV}$, data taken from the PDG

Furthermore, the neutral isospin partner for the $Z_{c}(3900)$ has been observed be BESIII (see Fig. 4 right, preliminary result) in $e^{+} e^{-} \rightarrow \pi^{0} \pi^{0} J / \psi$. Here, the invariant mass of $\pi^{0} J / \psi$ pairs is plotted, exhibiting a structure with mass and width consistent with the corresponding parameters of $Z_{c}(3900)$. Thus, we conclude that an isospin triplet has been observed.

Similar structures have been observed[9] at BESIII by looking at the invariant mass distributions of $\pi^{ \pm} h_{c}$ and $\pi^{0} h_{c}$ in $e^{+} e^{-} \rightarrow \pi^{+} \pi^{-} h_{c}$ and $e^{+} e^{-} \rightarrow \pi^{0} \pi^{0} h_{c}$ (see Fig. 5), establishing a second isospin triplet Z(4020). A remarkable feature of Z(3900) and Z(4020) is their location in the vicinity of
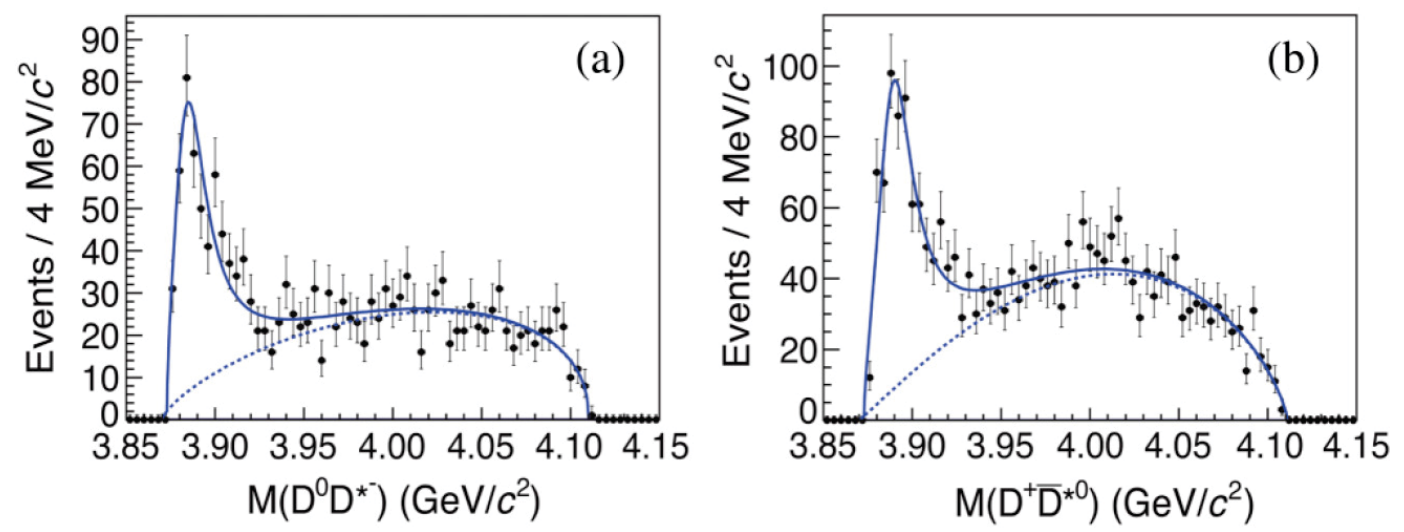

Figure 7. Invariant mass distributions for $D^{0} \bar{D}^{*-}$ (a) and $D^{+} \bar{D}^{* 0}$ (b) from $e^{+} e-\rightarrow \pi D \bar{D}^{*}$ at $\sqrt{s}=4260 \mathrm{GeV}$. The solid curves in Fig. 2 show the fit results and the dashed curves show the nonresonant background

various open charm thresholds. Fig. 6 shows a compilation of the thresholds for decays into pairs of open charm mesons. Thus, it is interesting to search for the present of structures in the decays to 
channels with pairs of open charm mesons . BESIII has observed[10]a charged $\left(D \bar{D}^{*}\right)^{ \pm}$mass peak in

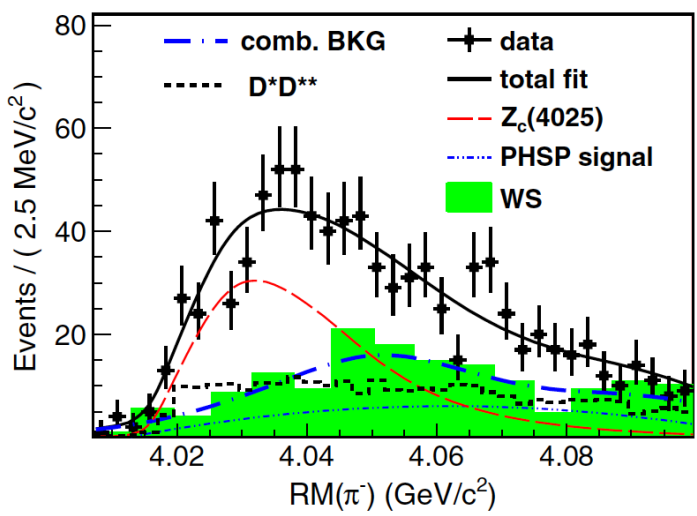

Figure 8. $\pi^{-}$recoil mass spectrum from $e^{+} e-\rightarrow \pi^{ \pm}\left(D * \bar{D}^{*}\right)^{\mp}$ at $\sqrt{s}=4260 \mathrm{GeV}$. The solid curves show the results of an unbinned maximum likelihood fit and the dashed curves show the nonresonant background

$e^{+} e-\rightarrow \pi D \bar{D}^{*}$ at $\sqrt{s}=4260 \mathrm{GeV}$. Fig 7 shows the corresponding invariant mass distributions for $D^{0} \bar{D}^{*-}$ (a) and $D^{+} \bar{D}^{* 0}(\mathrm{~b})$.

When fitted to a mass-dependent-width Breit-Wigner line shape, the pole masse and width are determined to be $(3883.9 \pm 1.5($ stat $) \pm 4.2($ syst $)) M e V / \mathrm{c}^{2}$ and a width of $24.8 \pm 3.3($ stat $) \pm 11($ syst $\left.)\right)$ $\mathrm{MeV}$. Within the errors, these values are consistent with the parameters of $\mathrm{Z}(3900)$, leading to the speculation that both charged charmonium - like structures might be of identical origin.
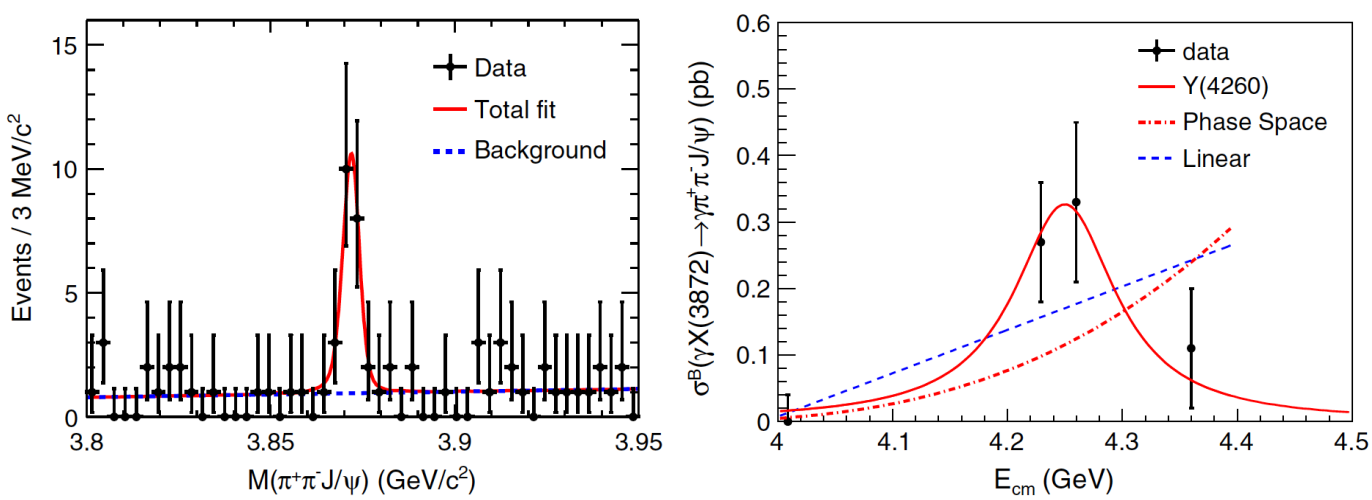

Figure 9. (left): Invariant mass distribution of $\pi^{+} \pi^{-} J / \psi$ for the process $e^{+} e^{-} \rightarrow \pi^{+} \pi^{-} J / \psi$ at $\sqrt{s}=4260 \mathrm{GeV}$. (right) extracted $\mathrm{X}(3872)$ cross section as a function of $\sqrt{s}$ in the region of the $\mathrm{Y}(4260)$ resonance. For details, see Ref. [12].

Furthermore, a charged structure in the $\left(D * \bar{D}^{*}\right)^{ \pm}$mass system has been found which we label with $Z(4025)$ [11] with mass and width parameters compatible with the $Z_{c}(4020)$. This is shown in Fig. 8, where the recoil mass distribution of charged pions from $e^{+} e-\rightarrow \pi^{ \pm}\left(D * \bar{D}^{*}\right)^{\mp}$ at $\sqrt{s}=4260$ $\mathrm{GeV}$ is plotted.

Finally, BESIII observed a radiative transition $Y(4260) \rightarrow X(3872)$ [12]. We have investigated the process $e^{+} e^{-} \rightarrow \gamma \pi^{+} \pi^{-} J / \psi$ in the vicinity of the Y(4260) resonance. Fig 9 (left) shows the invariant mass distribution of the $\pi^{+} \pi^{-} J / \psi$ system. A clear signal for $\mathrm{X}(3872)$ is observed, with a statistical significance of $6.3 \sigma$. As Fig. 7 (right) indicates, the data is consistent with the assumption of a 
radiative decay of $Y(4260) \rightarrow X(3872)$. This result provides for the first time a connection between two exotic XYZ states, the $Y(4260)$ and the $X(3872)$ via a radiative transition.

\section{Summary and outlook}

Charmonium spectroscopy is an excellent probe to study QCD in the transition region between the perturbative and non-perturbative regime. About 40 years after the discovery of the $J / \psi$, this field has experienced a strong revival, driven by the discovery of new charmonium-like states with unexpected features not fitting in the predicted charmonium spectrum above the open charm threshold. Recently, new charged charmonium-like structures have been discovered by BESIII and their structure is still subject of extensive research, both experimentally and theoretically.

For the future, it will be crucial to further explore the systematics of the new XYZ states, establishing their quantum numbers and observing hadronic and radiative transitions between these states, to conventional charmonium states or even to states without hidden charm.

Of particular importance is the clarification of the internal structure of these states. Did we discover new types of hadrons (tetra-quarks, hybrids), are we observing molecular structures formed by binding two color-neutral objects or are we encountering dynamical effects producing structures in invariant mass distributions that should not be identified as particles?

The currently running experiments with access to this field (BESIII, LHCb) and the future experiments (Belle II, PANDA) will hopefully solve the puzzle of the XYZ states, together with refined models and more fundamental calculations with predictive power. After all, QCD is one of the basic interactions in the Standard Model and we cannot claim that we have understood the strong interaction if we are not able to predict essential features of the observed hadron spectrum.

\section{Acknowledgement}

The BESIII collaboration thanks the staff of BEPCII and the computing center for their strong support. This work is supported in part by the Ministry of Science and Technology of China under Contract No. 2009CB825200; Joint Funds of the National Natural Science Foundation of China under Contracts Nos. 11079008, 11179007, U1332201; National Natural Science Foundation of China (NSFC) under Contracts Nos. 10625524, 10821063, 10825524, 10835001, 10935007, 11125525, 11235011; the Chinese Academy of Sciences (CAS) Large-Scale Scientific Facility Program; CAS under Contracts Nos. KJCX2-YW-N29, KJCX2-YW-N45; 100 Talents Program of CAS; German Research Foundation DFG under Contract No. Collaborative Research Center CRC-1044; Istituto Nazionale di Fisica Nucleare, Italy; Ministry of Development of Turkey under Contract No. DPT2006K-120470; U. S. Department of Energy under Contracts Nos. DE-FG02-04ER41291, DE-FG02-05ER41374, DE-FG02-94ER40823, DESC0010118; U.S. National Science Foundation; University of Groningen $(\mathrm{RuG})$ and the Helmholtzzentrum fuer Schwerionenforschung GmbH (GSI), Darmstadt; WCU Program of National Research Foundation of Korea under Contract No. R32-2008-000-10155-0.

\section{References}

[1] S.-K. Choi et al. (Belle Collaboration), Phys. Rev. Lett. 91.262001 (2003)

[2] B. Aubert at al. (BaBar Collaboration), Phys. Rev. Lett. 95, 142001 (2005)

[3] R. Aaij et al. (LHCb Collaboration), Phys. Rev. Lett. 110, 222001 (2013)

[4] G.T. Bodwin et al., arXiv:1307.7425 [hep-ph]

[5] Nucl. Instrum. Meth. A 614, 345 (2010) 
[6] M. Ablikim et al. (BESIII Collaboration), Phys. Rev. Lett. 110, 252001 (2013)

[7] Z. Q. Liu et al. (Belle Collaboration), Phys. Rev. Lett. 110, 252002 (2013)

[8] T. Xiao et al., Phys. Lett. B727, 366 (2013)

[9] M. Ablikim et al. (BESIII Collaboration), arXiv:1409.6577 [hep-ph], submitted to Phys. Rev. Lett.

[10] M. Ablikim et al. (BESIII Collaboration), Phys. Rev. Lett. 112, 022001 (2014)

[11] M. Ablikim et al. (BESIII Collaboration), Phys. Rev. Lett. 112, 132001 (2014)

[12] M. Ablikim et al. (BESIII Collaboration), Phys. Rev. Lett. 112, 092001 (2014) 